\title{
Research Progress of Continuous Blood Purification in the Treatment of Severe Sepsis in Children
}

\author{
Weikai Wang ${ }^{1 \#}$, Yun Du ${ }^{2 \#}$, Aiqin Cheng ${ }^{3}$, Shunli Liu ${ }^{4}$, Lin Wei ${ }^{1}$, Jianna Li ${ }^{1}$, Yirong Wang ${ }^{1 *}$, Li Wang ${ }^{*}$ \\ ${ }^{1}$ Department of PICU, Gansu Provincial Maternity and Child-care Hospital, Lanzhou 730050, Gansu Province, China \\ ${ }^{2}$ Department of Pediatrics, Lintao County Hospital of Traditional Chinese Medicine, Lanzhou 730050, Gansu Province, \\ China
}

${ }^{3}$ Department of Pediatrics, Wushan County Hospital, Wushan 741300, Gansu Province, China

${ }^{4}$ Department of Pediatrics, Yongdeng County Hospital, Yongdeng 730300, Gansu Province, China

${ }^{5}$ Department of Pediatrics, Baiyin Central Hospital, Baiyin 730913, Gansu Province, China

\#Weikai Wang and Yun Du both are the first authors and contributed equally to this work.

*Yirong Wang and Li Wang both are the corresponding authors.

\begin{abstract}
Pediatric sepsis is the most common disease in pediatric critical illness, because the main reason for the disease is that children's immune level is not high or the immune system is not perfect, when children's lung, abdominal cavity and blood system are infected, it will cause systemic inflammation and immune dysfunction. Early clinical symptoms are mainly irregular and intermittent fever. When the disease develops to severe sepsis, the children will suffer from acute heart failure, oliguria, respiratory alkalosis and even multiple organ failure. The incidence of death is high. It is reported that the incidence rate of sepsis in children can reach $0.3 \%$, and the mortality rate is $50 \%$. High incidence rate, high mortality rate and high treatment cost are the biggest problems in the pediatric field. In the past, the clinical hope of clearing away heat and toxin, promoting blood circulation and removing stasis, strengthening inflammation and other methods in traditional Chinese medicine, but the treatment effect is not ideal. With the improvement of modern medical understanding of sepsis, continuous blood purification therapy is introduced into the treatment of children with severe sepsis. In order to further explore the effect of continuous blood purification in the treatment of children with severe sepsis, the author summarizes the clinical practice experience and relevant literature, hoping to provide reference for relevant medical staff.
\end{abstract}

Key words: Continuous blood purification; Severe sepsis in children; Inflammatory factors; Lactate level

Publication date: May, 2021; Publication online: 31 May, 2021

*Corresponding author: Yirong Wang, Li Wang, 35487284@qq.com

\section{Introduction}

Severe sepsis refers to sepsis complicated with multiple organ dysfunction, hypoperfusion or hypotension, which leads to insufficient organ perfusion in children and increases the risk of death. Therefore, timely and effective treatment for children with severe sepsis is very important.
At present, in the clinical treatment of children with severe sepsis, comprehensive treatment such as anti-infection and electrolyte correction is often used, but the curative effect is not ideal. With the application of continuous blood purification treatment, it can continuously remove inflammatory substances in children's body, reduce the level 
of inflammatory factors, and reduce its damage to the body organs. Xin Keke ${ }^{[1]}$ in its clinical research, through the two groups of children were treated with conventional therapy and continuous blood purification therapy, and then compared the clinical efficacy of the two groups of children. It is found that continuous blood purification can promote the stability of hemodynamics, regulate the level of inflammatory factors and shorten the treatment time in children with severe sepsis; Liang Silong ${ }^{[2]}$ and others found that the blood purification treatment scheme for children with severe sepsis was effective as high as $93.75 \%$ through the changes of inflammatory factors in the two groups of children after routine treatment and continuous blood purification treatment, and the effect was significant, which could effectively remove the inflammatory mediators in the body of children, effectively improve the internal environment of children, and improve the rehabilitation efficiency, which was widely used in clinical application has high value. Through consulting a large number of clinical research data, the author summarizes the research ideas of continuous blood purification in the treatment of children with severe sepsis, hoping that through this study, it can play a certain reference for pediatric emergency field in the treatment of children with severe sepsis.

\section{Understanding of sepsis in modern medicine}

The incidence rate of severe sepsis is high and mortality rate is higher. It is the most common systemic inflammatory response syndrome in pediatric ICU wards. The main pathophysiological basis of this disease is the large number of trauma, burns, infection, shock, and the inflammatory cell factors caused by large surgical operations, which leads to multiple organ dysfunction in children. In the past 20 years, the medical circles at home and abroad pay special attention to the study of this disease. It is found that the pathogenesis of severe sepsis is extremely complex, which is closely related to the pathophysiological changes of multiple systems and organs. At present, the research has been involved in a series of fields, such as intestinal endotoxin translocation, staphylococcal exotoxin and its pathogenic effect, signal transduction mechanism, inflammatory response and immune paralysis, coagulation dysfunction, etc. with the deepening of the research, relatively efficient treatment schemes have been applied in clinic, and the application effect is obvious, among which the most mature treatment scheme is continuous blood transfusion Purification therapy ${ }^{[3]}$.

\section{Mechanism of continuous blood purification in the treatment of children with severe sepsis}

\subsection{Regulate blood lactate, troponin and coagulation function}

Combined with clinical diagnosis data, lactic acid, troponin, and coagulation state of children with severe sepsis showed abnormal expression. The only standard to measure the body tissue perfusion level and blood oxygen index of children is the level of lactic acid, and the serum index that can reflect the degree of myocardial injury in children is troponin specific index. When severe sepsis occurs in children, inflammatory factors and free radicals in the body will be released in large quantities, cell membrane permeability will be enhanced, and pyrolytic troponin will be released into the blood. Through blood monitoring, high levels of troponin can clearly reflect the severity of heart function damage in children. The results of clinical studies show that the levels of D-dimer, prothrombin time, partially activated thromboplastin time and thrombin time can reflect the severity of the disease. Therefore, the mortality rate of children with severe sepsis complicated with low coagulation and high bleeding tendency is higher.

When severe sepsis children's body inflammation is strong, their microcirculation and tissue perfusion will be in a poor level, the body tissue will be seriously lack of oxygen supply, metabolic imbalance, induce abnormal lactic acid metabolism, therefore, one of the most obvious indicators of severe sepsis is high blood lactate value level. In the course of treatment, if the lactate level of children can be effectively inhibited and gradually decreased, the condition will gradually improve; The clinical indicators of those children with disease death are mostly due to the body's inflammatory stress can not be effectively contained, liver function tends to fail, gradually losing the ability to clear lactic acid, resulting in the continuous increase of lactic acid level in the body, the body will be in a vicious circle, the body organs continue to damage, and gradually fail.

TNT and TnI in serum troponin are the most important clinical indicators to reflect the immune system level of children with severe sepsis. They have high sensitivity to reflect the myocardial injury of children. Scholars such as $\mathrm{Li}$ Yumei ${ }^{[4]}$ believe that the myocardial tolerance to oxygen debt is poor. If the inflammatory state of children is strong, the tissue oxygen supply will be seriously unbalanced, and the blood lactic acid value will continue to rise, which will aggravate the degree of myocardial damage, and then lead to 
the increase of the expression level of myocadherin, which indicates that children with severe sepsis generally have severe myocardial damage If the condition can be better controlled, the myocardial injury will be improved, otherwise, the condition will be further aggravated, threatening the life safety of children.

Coagulation dysfunction is the most obvious clinical index in children with severe sepsis, especially PT, APTT, D-D and other indexes in blood test are significantly increased, which is related to the cascade reaction of inflammatory mediators triggered by the invasion of pathogenic microorganisms and secretion of internal and external toxins. When the inflammatory mediators act on the capillary endothelium, the anticoagulant substances in the body of children are reduced, and the fibrinolytic system is damaged, which makes the coagulation process difficult ${ }^{[5-6]}$.

From the clinical analysis of the survival of children with severe sepsis, with the further treatment, the blood lactic acid, troponin, and coagulation state of children will gradually improve. The clinical treatment of children with severe sepsis pointed out the direction, that is, through continuous blood purification, the toxic substances in children's body can be completely removed, the blood lactic acid and troponin levels can be adjusted, the coagulation state can be improved, and the internal environment stability and physiological balance can be promoted, which can provide favorable conditions for further treatment ${ }^{[7-9]}$.

\subsection{Reduce the expression of inflammatory factors}

Inflammatory factors are the key factors for sepsis to develop into severe sepsis. In the early stage of sepsis, the inflammatory effector cells in children's body will automatically recognize the pathogen antibody and promote the body to release a large number of inflammatory factors. With the increase of the secretion of inflammatory factors, the inflammatory factors will continue to release, which will lead to the damage of immune function in children, seriously affect the expression of hemodynamic indicators, and further aggravate the disease The disease developed into severe sepsis. Therefore, reducing the expression of inflammatory factors is the core of the treatment of severe sepsis.

TNF- $\alpha$ is a mononuclear pro-inflammatory factor, which is the primary mediator of inflammatory response. When the level of TNF- $\alpha$ in children's serum increases, the blood vessel tension and cardiac contractility will decrease sharply, the vascular permeability will increase, and the inflammatory reaction state of the body will be very strong.
Continuous blood purification can effectively inhibit the release level of serum TNF- $\alpha$ and regulate the immune response, which has a positive role in promoting the prognosis of severe sepsis.

Continuous blood purification mainly removes inflammatory substances and other toxic substances in children's serum through diffusion, convection and adsorption, so as to reduce the level of inflammatory factors and effectively alleviate the damage of inflammatory reaction to various organs of the body ${ }^{[11]}$. Among them, dispersion is mainly to transport the semipermeable membrane of blood filter from the high concentration end to the low concentration end under the effect of solute concentration difference. Because molecules will move disorderly in this process, small molecular solutes such as creatinine, urea nitrogen and uric acid can be quickly removed from the blood; However, the convection is that under the action of water transmembrane pressure, the blood flows from the high pressure end to the low pressure end when passing through the filter, and all the inflammatory factors in the blood are taken out of the body; Adsorption is that solute is adsorbed on the surface of semipermeable membrane to filter toxic substances in blood. It is only related to the adsorption area of semipermeable membrane, and will not be affected by the size and density of solute. However, this process emphasizes the continuity, only through continuous purification, can we completely remove the collective inflammatory factors and other toxic substances, achieve the metabolic balance of patients, and achieve the purpose of improving the condition ${ }^{[12]}$.

\subsection{Serum procalcitonin level decreased}

Gamma globulin combined with continuous blood purification can effectively reduce the level of serum procalcitonin in children, protect children's organs from damage, and accelerate the recovery speed. Gamma globulin contains all kinds of antibodies needed in human serum, which can significantly improve the resistance of children and enhance the ability of anti-infection ${ }^{[13-15]}$. After injecting gamma globulin into children, a large amount of antibody is injected to neutralize the virus and toxin in children's body, and even kill them all, so as to improve the immune system of children.

Serum procalcitonin (PCT) is a kind of protein that can reflect the active degree of systemic inflammatory response. The level of PCT will rise in the early stage of systemic inflammatory response. The level of PCT is related to the 
type of infection, the type of bacteria and the severity of immune response. Gamma globulin combined with continuous blood purification can significantly reduce the level of serum procalcitonin, avoid organ damage and immune system disorder in children, and then improve the effect of disease control, which has an important impact on the prognosis and prognosis of children ${ }^{[16]}$.

\section{Conclusion}

In conclusion, continuous blood purification for the treatment of children with severe sepsis, through the diffusion, convection and adsorption of three ways to remove toxins in the body of children, the curative effect is exact, can promote the stability of hemodynamics in children, reduce the levels of inflammatory factors, blood lactic acid, serum troponin and serum procalcitonin, and improve the coagulation function, accelerate the recovery of children It can be used as an effective treatment in clinical popularization.

\section{References}

[1] Xin KK. Application effect of continuous blood purification in patients with severe sepsis [J]. Journal of Community Medicine, June 20, 2018

[2] Liang SL. Clinical effect of continuous blood purification on 48 children with severe sepsis [J]. World's Latest Medical Information Digest, July 26, 2019

[3] Liu WL. Effect of continuous blood purification on inflammatory factors and hemodynamics in patients with severe sepsis [J]. Tibet Medicine, 2020-04-01

[4] Li YM, Chen CD, Zhang DR, et al. Dynamic changes of blood lactic acid, troponin and coagulation indexes in children with severe sepsis and their relationship with prognosis $[\mathrm{J}]$. Chinese Journal of Nosocomial Infection, 2020-11-30.

[5] Dai WL, Huang HF. Efficacy of continuous blood purification in the treatment of severe sepsis [J]. World's Latest Medical Information Digest, January 15, 2019.

[6] Liu N, Zhang ZP. Analysis of the efficacy of continuous blood purification in the treatment of severe sepsis $[\mathrm{J}]$. Electronic Journal of Cardiovascular Disease of Integrated Traditional Chinese and Western medicine, November 28, 2018
[7] Shi L, He Y, Li K. Clinical effect of ulinastatin + continuous blood purification on 40 cases of severe sepsis [J]. Famous Doctor, 2019 (2): 238.

[8] Dai WL, Huang HF. Observation on the efficacy of continuous blood purification in the treatment of severe sepsis [J]. World's Latest Medical Information Abstracts, 2019, 19 (5): 9-10.

[9] Huo ZR, Yang SJ. Clinical study of Shenqi Fuzheng Injection Combined with continuous blood purification in the treatment of severe sepsis [J]. Journal of Modern Integrated Traditional Chinese and Western Medicine, 2017, 26(7): 756-758.

[10] Chen J. The effect of volume overload on the prognosis of children with sepsis and the effect of diuretics [D]. Suzhou University, 2017.

[11] Zhang CX, Wang JC, Chao LM, et al. Effect of gamma globulin combined with continuous blood purification on serum procalcitonin and critical score in children with severe sepsis $[\mathrm{J}]$. Modern Biomedical Exhibition, 2016, 16 (35).

[12] Cai LF, Zhang JJ, Dong JX, et al. Analysis of the efficacy of continuous blood purification in the treatment of severe sepsis with stress cardiomyopathy [J]. Chinese Journal of medicine, 2016, 18 (5): 458-459

[13] Lu XN, Liu H, Zhou FH. Efficacy analysis of continuous blood purification in the treatment of severe sepsis [J]. Journal of Chinese PLA Medical College, 2014, 35 (11): 1090-1092 + 1100

[14] Jiang BJ, Wang $\mathrm{CH}$, Peng $\mathrm{H}$, et al. Clinical study of continuous blood purification in the treatment of severe sepsis with ARDS [J]. Journal of Tongji University (Medical Edition), 2014, 35 (2): 77-81 + 93.

[15] Chen ZJ. Efficacy of high flux hemofiltration in children with severe sepsis [D]. Zhejiang University, 2013

[16] Yu AJ. Therapeutic effect of early continuous blood purification on severe sepsis with intra-abdominal hypertension [C]. Critical Care Medicine Branch of Zhejiang Medical Association. First West Lake Critical Care Medicine Forum and 2011 Zhejiang Critical Care Medicine Academic Annual Meeting. Critical Care Medicine Branch of Zhejiang Medical Association: Zhejiang Science and Technology Association, 2011: 475-476. 